\title{
ON VACUUM-ENERGY DECAY FROM PARTICLE PRODUCTION
}

\author{
F.R. Klinkhamer \\ Institute for Theoretical Physics, Karlsruhe Institute of Technology (KIT), \\ 76128 Karlsruhe, Germany \\ frans.klinkhamer@kit.edu
}

\begin{abstract}
A simplified (but consistent) description of particle-production backreaction effects in de Sitter spacetime is given.

Journal: Mod. Phys. Lett. A 27, 1250150 (2012)

Preprint: arXiv:1205.7072

Keywords: Quantum field theory in curved spacetime, early universe, cosmological constant, dark energy.

PACS: 03.70.+k, 98.80.Cq, 98.80.Es, 95.36.+x
\end{abstract}

\section{Introduction}

Polyakov has argued in a recent series of papers $12 \mid 3$ that de Sitter spacetime suffers from an explosive production of particles, if these particles have nonvanishing selfinteractions. He did not address quantitatively the issue of backreaction, namely, how the original de Sitter spacetime is changed by the produced particles. Here, we offer a few heuristic remarks on this issue, awaiting the definitive calculation of the relevant effective parameters. Incidentally, an extensive list of references on the problem of vacuum-energy decay can be found in the original papers $\frac{1213}{3}$ and a useful follow-up paper ${ }^{4}$ (see also Refs. $[5$ and 6 for two early papers).

In four-dimensional de Sitter (dS) spacetime ${ }^{7 / 8}$ with a positive cosmological constant $\Lambda$, infrared higher-loop quantum effects are also expected to lead to instability of the pure (matter-less) vacuum state. ${ }^{2}$ With the macroscopic timescale being set by the inverse of the Hubble constant $H_{\mathrm{dS}}$ and $\Lambda$ being the only macroscopic energy-density scale available, the produced energy density of particles is given by the following expression $(c=\hbar=1)$ :

$$
\begin{aligned}
\left.\dot{\rho}_{M}\right|_{\mathrm{dS}, \rho_{M}=0} & =\sqrt{3} \gamma H_{\mathrm{dS}} \Lambda, \\
H_{\mathrm{dS}} & \equiv \sqrt{\Lambda /\left[3\left(E_{P}\right)^{2}\right]}, \quad \Lambda>0, \\
E_{P} & \equiv 1 / \sqrt{8 \pi G_{N}}, \quad G_{N}>0,
\end{aligned}
$$

where the square-root factor on the right-hand side of (1a) has been added for later convenience and the dimensionless parameter $\gamma$ is a short-hand notation for 
$\gamma_{V}$ (another parameter $\gamma_{M}$ will be introduced subsequently). The parameter $\gamma>0$ involves the scalar self-coupling constants (see further comments below) and the overdot on the left-hand side of (1a) stands for differentiation with respect to a cosmic time coordinate $t$ to be defined later (the resulting matter perturbation breaks the original de Sitter symmetry). It needs to be emphasized, right from the start, that Polyakov's tentative result (1a) solely relies on the careful study of quantum field theory in curved spacetime and does not require the introduction of new theories.

The constant growth rate from (1a) only holds as long as $\rho_{M} \ll \Lambda$, so that backreaction effects can be neglected. In order to address the further evolution $\left(\rho_{M} \sim \Lambda\right.$ ), we replace the cosmological constant $\Lambda$ by a dynamic vacuum energy density $\rho_{V}$. That is, we split the backreaction effects from the produced particles into a standard-matter-type component and a vacuum-type component.

\section{Dynamic Vacuum Energy}

For our purpose, the following classical action $\frac{910}{10}$ can be used:

$$
\begin{aligned}
S_{\text {class }} & =-\int d^{4} x \sqrt{-\operatorname{det}(g)}\left[\frac{1}{2}\left(E_{P}\right)^{2} R+\epsilon_{V}(q)+\mathcal{L}_{M}\right], \\
\mathcal{L}_{M} & =-\frac{1}{2}(\partial \phi)^{2}+\frac{1}{2} m^{2} \phi^{2}+\frac{1}{4} \kappa \phi^{4},
\end{aligned}
$$

where the matter part (2b) is entirely standard, consisting of a massive real scalar field $\phi(x)$ with quartic coupling constant $\kappa>0$. In (2a),$\epsilon_{V}(q)$ is a generic function of a $q$-type field, which is essentially a nonfundamental scalar field $q(x)$ with a constant nonzero expectation value $q_{0}$ in the equilibrium state. One particular realization of $q(x)$ is by use of a three-form gauge field $A(x)$; see Refs. 9 and 10 for details and original references.

The vacuum energy density in the corresponding gravitational field equation is denoted by $\rho_{V}$ and differs, in general, from the energy density $\epsilon_{V}$ entering the action. In terms of a single $q$-type field, the gravitating vacuum energy density takes the following form $\frac{910}{}$ :

$$
\rho_{V}(q)=-P_{V}(q)=\epsilon_{V}(q)-q \frac{d \epsilon_{V}(q)}{d q} .
$$

Henceforth, we assume that $\rho_{V}$ takes sub-Planckian values and that the scalar particles are sufficiently heavy, 2

$$
\begin{aligned}
& 0<\rho_{V} \ll\left(E_{P}\right)^{4} \approx\left(2.44 \times 10^{18} \mathrm{GeV}\right)^{4}, \\
& m \gg \sqrt{\rho_{V} /\left(E_{P}\right)^{2}} .
\end{aligned}
$$

Condition (4a) allows us to rely on classical gravity. In the cosmological context to be discussed shortly, condition (4b) implies that the "response-time" of the particle 
wave function is very much less than the relevant cosmological timescale. Condition (4b) in the cosmological context also suggests that the produced scalar particles are nonrelativistic, their rest mass being very much larger than the Gibbons-Hawking temperature $^{8}$ of the corresponding de Sitter spacetime, $m \gg T_{\mathrm{GH}}=H_{\mathrm{dS}} / 2 \pi$.

The action (2) is invariant under general coordinate transformations or, more precisely, diffeomorphisms. Diffeomorphism invariance implies energy-momentum conservation of the matter component (cf. Appendix E.1 of Ref. 11). Here, the matter component is given by the dynamic vacuum energy density $\rho_{V}=-P_{V}$ and the scalar field $\phi$.

\section{FRW Equations}

Now let us restrict ourselves to a spatially flat Robertson-Walker metric (in standard comoving coordinates 5111 with cosmic time $t$ ), a homogeneous pressurelessperfect-fluid component $\left(w_{M} \equiv P_{M} / \rho_{M}=0\right)$, and a homogeneous vacuum-energy component $\left(w_{V} \equiv P_{V} / \rho_{V}=-1\right)$. Then, the Friedmann-Robertson-Walker (FRW) equations [in dimensionless form given by (6c) and (6d) below] are consistent only when using the following generalization of (1a):

$$
\dot{\rho}_{M}+3 H \rho_{M}=\gamma \sqrt{\left|\rho_{V}\right| /\left(E_{P}\right)^{2}} \rho_{V},
$$

and the following vacuum-energy-density equation:

$$
\dot{\rho}_{V}=-\gamma \sqrt{\left|\rho_{V}\right| /\left(E_{P}\right)^{2}} \rho_{V},
$$

where the equation of state $P_{V}=-\rho_{V}$ has been used on the left-hand side. The absolute value $\left|\rho_{V}\right|$ has been employed in the square-roots on the right-hand sides of (5), but this is, strictly speaking, not necessary if (4a) holds.

Before presenting the complete set of differential equations, let us comment on the physical interpretation of (5a) and (5b). The first set of comments concerns the starting point, the de Sitter-spacetime calculation giving the right-hand sides of (1a) and (5a). Given the theory (2), the dimensionless parameter $\gamma>0$ involves expressions containing positive powers of the quartic coupling constant $\kappa$. The main results of Polyakov's investigations are, first, that higher-loop quantum corrections may contribute the seed for $\gamma$ (for example, from a 1-loop Feynman diagram implicit in the second paragraph of Sec. 5 in Ref. 2 but not evaluated explicitly) and, second, that a chain-reaction-type evolution can amplify microscopic effects to macroscopic ones [see Eq. (27) in Ref. 2 for an example of this explosive behavior and Sec. 5 for further discussion]. Both results are infrared effects, with the whole of de Sitter spacetime contributing.

The second set of comments concerns the backreaction. Purely from the classical theory (2), the right-hand sides of (5a) and (5b) should vanish: see, respectively, 
Eqs. (4.6) and (4.5) in Ref. 10 for the case of a constant gravitational coupling parameter $G=G_{N}$. In the stationary background of the classical de Sitter spacetime and with classical $q$-type fields entering the vacuum energy density $\rho_{V}$, the scalar quantum field theory corresponding to (2b) would give rise to particle production from infrared quantum effects 213 as given by (1a). In the dynamic context [timedependent vacuum energy density $\left.\rho_{V}(t)\right]$, this results in the expression shown on the right-hand side of (5a), with the opposite expression on the right-hand side of (5b) from energy-momentum conservation (tracing back to the diffeomorphism invariance of the theory, including quantized-matter effects). There are further terms on the right-hand-sides of (5a) and (5b), which are of the form $\pm \gamma_{M} \sqrt{\left|\rho_{V}\right| /\left(E_{P}\right)^{2}} \rho_{M}$ for $\gamma_{M}>0$ and which correspond to a type of stimulated emission of matter particles (cf. Sec. 5 in Ref. 2). These terms are the outcome of the chain-reaction-type evolution mentioned in the previous paragraph but will, for the moment, not be considered explicitly $\left(\gamma_{M}=0\right)$.

As promised, we give the complete set of ordinary differential equations (ODEs), obtained by adding the two FRW equations which follow from the action (2a) for the spatially flat $(k=0)$ Robertson-Walker metric. Introducing dimensionless variables by use of appropriate powers of $E_{P}$, these ODEs are

$$
\begin{aligned}
\dot{r}_{V} & =-\gamma\left|r_{V}\right|^{1 / 2} r_{V}, \\
\dot{r}_{M}+3 h r_{M} & =+\gamma\left|r_{V}\right|^{1 / 2} r_{V}, \\
2 \dot{h} & =-r_{M}, \\
3 h^{2} & =r_{V}+r_{M},
\end{aligned}
$$

with the dimensionless Hubble parameter $h(\tau)$ and the dimensionless cosmic time $\tau$ (the overdot now stands for differentiation with respect to $\tau$ ). The dimensionless variable $r_{M}(\tau) \geq 0$ corresponds to the standard-matter energy density $\rho_{M}(t) \geq 0$ with constant equation-of-state parameter $w_{M}=0$. Similarly, $r_{V}(\tau)=-p_{V}(\tau)$ corresponds to $\rho_{V}(t)=-P_{V}(t)$. Elaborating on the previous discussion of energymomentum conservation, the differential system (6) is seen to be consistent: (6c) follows from taking the time derivative of (6d) and using (6a) and (6b).

In order to match the setup of (1a), the boundary conditions for the ODEs (6a), (6b), and (6c) are taken as follows:

$$
\begin{aligned}
r_{V}(1) & =\lambda>0, \\
r_{M}(1) & =0, \\
h(1) & =\sqrt{\lambda / 3},
\end{aligned}
$$

with the Friedmann equation (6d) acting as a constraint. The boundary conditions 

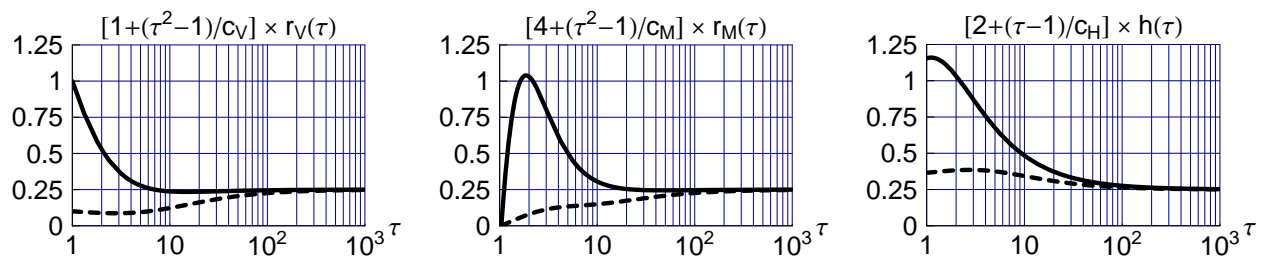

Fig. 1. Numerical solution of the ODEs (6) for vacuum-energy decay parameter $\gamma=1$ with different boundary conditions (7), the upper (full) curves corresponding to an initial dimensionless cosmological constant $\lambda \equiv \Lambda /\left(E_{P}\right)^{4}=1$ and the lower (dashed) curves to $\lambda=1 / 10$. The curves are rescaled by constant numerical factors at $\tau=1$ and by appropriate $\tau$ monomials for $\tau \gg 1$. The coefficients $\left\{c_{V}, c_{M}, c_{H}\right\}$ used in this rescaling are defined in (10) and take the numerical values $\{16,12.28,6.14\}$. The model universe for $\tau \sim 1$ resembles a segment of de Sitter spacetime (8) with approximately constant Hubble parameter, $h(\tau) \sim h(1)=\sqrt{\lambda / 3}$. As $\tau$ increases, the vacuum energy density $r_{V}(\tau)$ drops from the value $\lambda$ to zero and the model universe approaches Minkowski spacetime with $h=0$.

are, therefore, characterized by the single number $\lambda \equiv \Lambda /\left(E_{P}\right)^{4}$ and the ODEs (6) by the single model-parameter $\gamma$.

\section{Exact $k=0$ FRW Solution}

For $\gamma=0$, the solution of the ODEs (6) with boundary conditions (77) corresponds to the standard (eternal) de Sitter spacetime, ${ }^{7}$ having the following constant functions:

$$
\left[\left\{r_{V}(\tau), r_{M}(\tau), h(\tau)\right\}\right]^{(\gamma=0)}=\{\lambda, 0, \sqrt{\lambda / 3}\}
$$

For $\gamma \rightarrow \infty$, the solution rapidly approaches the standard matter-dominated FRW solution (see the asymptotic result below). For $\gamma=1$, the numerical solution is shown in Fig. 1 .

Given the relative simplicity of the differential system (6) and boundary conditions (7), it is also possible to obtain the exact solution for $\gamma>0$,

$$
\begin{aligned}
{\left[r_{V}(\tau)\right]^{(\gamma>0)}=} & \left(\frac{1}{1 / \sqrt{\lambda}+\gamma(\tau-1) / 2}\right)^{2}, \\
{\left[r_{M}(\tau)\right]^{(\gamma>0)}=} & 3\left([h(\tau)]^{(\gamma>0)}\right)^{2}-\left[r_{V}(\tau)\right]^{(\gamma>0)}, \\
{[h(\tau)]^{(\gamma>0)}=} & \frac{1}{\kappa+\theta} \\
& \times \frac{(2 / \gamma)\left[(\kappa+\theta)^{\eta}-\kappa^{\eta}\right]+\sqrt{1 / 3}\left[(\eta+1)(\kappa+\theta)^{\eta}+(\eta-1) \kappa^{\eta}\right]}{\sqrt{3}\left[(\kappa+\theta)^{\eta}-\kappa^{\eta}\right]+(\gamma / 2)\left[(\eta-1)(\kappa+\theta)^{\eta}+(\eta+1) \kappa^{\eta}\right]},
\end{aligned}
$$


with definitions

$$
\theta \equiv \tau-1, \quad \eta \equiv \sqrt{1+12 / \gamma^{2}}, \quad \kappa \equiv 2 /(\gamma \sqrt{\lambda}) .
$$

Incidentally, the $h$ solution (9c) results from a generalized Riccati equation, namely, $2 \dot{h}+3 h^{2}=r_{V}$ with $r_{V}$ from (9a). [For the case of relativistic matter $\left(w_{M}=1 / 3\right)$ and the same $r_{V}$ from (9a), the relevant generalized Riccati equation is $\dot{h}+2 h^{2}=$ $(2 / 3) r_{V}$, which can also be solved explicitly.] Remark that, for fixed $\lambda>0$ and $\tau \geq 1$, solution (9) reduces to (8) as $\gamma$ approaches 0 from above.

The vacuum-energy density (9a) is seen to drop to zero monotonically as $\tau$ runs from 1 to infinity (the left panel of Fig. 1 shows a rescaled $r_{V}$ ). The asymptotic (attractor) solution for $\gamma>0$ has, moreover, an equal time-dependence for the vacuum and matter components:

$$
\begin{gathered}
{\left[r_{V, \text { asymp }}(\tau)\right]^{(\gamma>0)}=\frac{1}{4} c_{V} \tau^{-2} \equiv\left(4 / \gamma^{2}\right) \tau^{-2},} \\
{\left[r_{M, \text { asymp }}(\tau)\right]^{(\gamma>0)}=\frac{1}{4} c_{M} \tau^{-2} \equiv \frac{2}{3}\left(1+\sqrt{1+12 / \gamma^{2}}\right) \tau^{-2},} \\
{\left[h_{\text {asymp }}(\tau)\right]^{(\gamma>0)}=\frac{1}{4} c_{H} \tau^{-1} \equiv \frac{1}{3}\left(1+\sqrt{1+12 / \gamma^{2}}\right) \tau^{-1} .}
\end{gathered}
$$

The asymptotic energy-density ratio depends only on the decay parameter $\gamma$ :

$$
\lim _{\tau \rightarrow \infty} r_{M} / r_{V}=\frac{1}{6}\left(\gamma^{2}+\gamma \sqrt{\gamma^{2}+12}\right)
$$

which goes as $(1 / 3) \gamma^{2}$ for $\gamma \gg 1$ and as $\sqrt{1 / 3} \gamma$ for $0 \leq \gamma \ll 1$. For $\gamma=\mathrm{O}(1)$, the ratio (11) is of order 1 .

\section{Discussion}

Two generalizations can be mentioned at this point. First, similar results for the asymptotic behavior are obtained if stimulated-emission-type terms $\mp \gamma_{M}\left|r_{V}\right|^{1 / 2} r_{M}$ are added to the right-hand sides of the ODEs (6a) and (6b) but, here, we keep $\gamma_{M}=0$ for simplicity.

Second, it is also possible to consider the de Sitter perturbation (5) to hold for the spatially-closed $(k=1)$ Robertson-Walker metric. ${ }^{77}$ The ODEs (6) are changed as follows: the left-hand side of (6c) picks up a term $-2 k / a^{2}$ and the left-hand side of (6d) a term $+3 k / a^{2}$, where $a=a(\tau)$ is the cosmic scale factor with Hubble parameter $h \equiv \dot{a} / a$. Numerical $k=1$ solutions have been obtained for the following boundary conditions at the "waist" of de Sitter spacetime (minimum distance between geodesic normals; cf. Fig. 16 in Ref. (7): $r_{V}(0)=\lambda>0, r_{M}(0)=h(0)=0$, and $a(0)=\sqrt{3 / \lambda}$. (See Appendix $\mathrm{A}$ for corresponding analytic results.) The behavior found numerically is different for $\gamma$ below or above a critical value $\gamma_{c}$ which depends on $\lambda$ [for example, $\gamma_{c} \approx 0.9215$ for $\lambda=1$ ]. For $\gamma<\gamma_{c}$, the $k=1$ numerical 
solution has the same asymptotics as the $k=0$ solution given by (10) [having, in particular, the same value for the energy-density ratio [11)], even though different regions of spacetime are covered. But, for $\gamma \geq \gamma_{c}$, the $k=1$ perturbation (5) affects the de Sitter universe in a fundamentally different way: a big-crunch singularity is produced in a finite amount of time, just as happens for the standard matterdominated $k=1$ FRW universe. The underlying microscopic physics determines which of the two types of perturbations is more appropriate, $k=0$ or $k=1$, and with which value of the decay constant $\gamma$.

Let us return to the original theory (6) with $\gamma>0, \gamma_{M}=0$, and $k=0$. Considering the vacuum energy density $r_{V}(\tau)$, it is then possible to estimate the cross-over time between the de Sitter-type behavior (8) and the FRW-type behavior (10a). More specifically, the "half-life" of the de Sitter universe [defined as the time needed to reduce an initial value $r_{V}=\lambda$ from (7) by a factor 1/2] is found to have the following parametric behavior from (9a):

$$
t_{\mathrm{dS}-\text { half-life }}=2(\sqrt{2}-1) \frac{1}{\gamma} \frac{E_{P}}{\sqrt{\Lambda}},
$$

where the dimensions have been restored and the right-hand side is, as expected from (1a), proportional to the inverse of the Hubble constant (1b). Remark that our use of the term "half-life" is not intended to imply an exponential decay, which is indeed not the case for (10a).

The half-life (12) is a direct manifestation of backreaction effects from the original de Sitter-spacetime particle-production (1a). For $\gamma=\mathrm{O}(1)$, this backreaction timescale would be of order $1 / H_{\mathrm{dS}} \sim E_{P} / \Lambda^{1 / 2}$. Such a timescale would

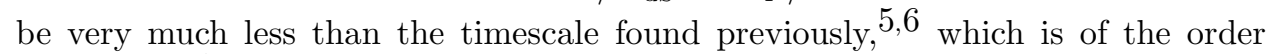
$\left[\left(E_{P}\right)^{4} / \Lambda\right]^{n / 2} 1 / H_{\mathrm{dS}}$ for $n \sim 1-2$ [recall the assumption that $\Lambda$ (or $\rho_{V}$ ) takes a value far below $\left.\left(E_{P}\right)^{4}\right]$. Physically, the short backreaction timescale (12) for $\gamma=\mathrm{O}(1)$ would be due to the chain-reaction effect mentioned in Sec. 3. This timescale of order $1 / H_{\mathrm{dS}}$ is indeed seen for the explicit solution of the simplified kinetic equation (27) in Ref. 2, assuming the relevant overlap integrals to be of order 1. (See also the respective Sections 5 and 6 of Ref. 4 for other examples without and with chain-reaction effects.)

Let us close with some further speculations. Assume that Polyakov's mechanism (1a) is relevant not only for the very early universe $\left(T \sim E_{P}\right)$ but also for the present epoch $(T \sim 3 \mathrm{~K})$. Then, purely phenomenologically, consider having a timedependent coupling $\gamma(\tau)$ in the differential system (6). If $\gamma(t)$ is like a step-function which drops to zero at a relatively recent moment $\left(t=t_{\text {freeze }}\right)$ in the history of the Universe, this would fix $\rho_{V}(t)$ at later times to the constant value $\rho_{V}\left(t_{\text {freeze }}\right)$. Given that the presently observed value of $\rho_{M} / \rho_{V}$ is approximately equal to $1 / 3$, this would suggest that the pre-freeze value of $\gamma$ should have been of order 1 . 
But it is also possible that the parameter $\gamma$ entering (1a) and (5) is significantly smaller than 1 and that the matter of the present Universe has a different origin (not solely the product of vacuum-energy decay).

Leaving these speculations aside, the clear priority at this moment is the de Sitter-spacetime calculation of the effective vacuum-energy decay parameters $\gamma_{V} \equiv \gamma, \gamma_{M}$, and others if present.

\section{Appendix A. Series-type $k=1$ FRW Solution}

The basic ODEs for perturbation (5) with $\gamma>0$ in a $k=1$ Robertson-Walker metric are

$$
\begin{aligned}
\dot{r}_{V}+\gamma\left|r_{V}\right|^{1 / 2} r_{V} & =0 \\
3(\dot{a} / a)^{2}+3 k / a^{2} & =r_{V}+r_{M}, \\
2 \ddot{a} / a+(\dot{a} / a)^{2}+k / a^{2} & =r_{V} .
\end{aligned}
$$

As discussed in Sec. [5 the boundary conditions can be taken as follows:

$$
r_{V}(0)=\lambda>0, \quad a(0)=\sqrt{3 / \lambda}, \quad \dot{a}(0)=r_{M}(0)=0 .
$$

With these boundary conditions, it is possible to study quantitatively the backreaction effects from vacuum-energy decay, for example, by imagining that $\gamma$ would be turned off for $\tau<0$ (corresponding to the standard de Sitter universe) and turned on for $\tau \geq 0$ (giving the perturbed de Sitter universe). Other boundary conditions give similar results.

Just as for the $k=0$ case in Sec. 4, the solution of A.1a) with boundary condition from (A.2) can be obtained immediately,

$$
r_{V}(\tau)=\lambda\left(\frac{1}{1+(\gamma / 2)(\sqrt{\lambda} \tau)}\right)^{2}
$$

With this $r_{V}(\tau)$, it remains to solve the single second-order ODE (A.1c).

Given that our interest is primarily in vacuum-energy decay for relatively small values of the decay constant $\gamma$ and cosmological constant $\lambda$, the following Ansatz turns out to be useful for small enough positive values of the cosmic time $\tau$ :

$$
a(\tau)=\sqrt{3 / \lambda} \cosh (\sqrt{\lambda / 3} \tau)\left[1+\gamma \sum_{n=2}^{\infty} c_{n}(\gamma)(\sqrt{\lambda} \tau)^{n}\right]
$$

where coefficient $c_{n}(\gamma)$ is a polynomial in $\gamma$. Inserting Ansatz (A.4) into the ODE (A.1c) gives the following Taylor expansion at $\tau=0$ :

$$
0=f_{0}\left(c_{2}\right)+f_{1}\left(c_{3}, c_{2}\right) \tau^{1}+f_{2}\left(c_{4}, c_{3}, c_{2}\right) \tau^{2}+\cdots,
$$


where the functions $f_{n}$ shown are linear in the leading coefficient $c_{n+2}$. It is, then, possible to solve sequentially for the coefficients $c_{n}$. The first eight coefficients are

$$
\begin{aligned}
& c_{2}=0, \quad c_{3}=-1 / 12, \quad c_{4}=\gamma / 32, \\
& c_{5}=\left(8-9 \gamma^{2}\right) / 720, \quad c_{6}=\gamma\left(-2+3 \gamma^{2}\right) / 576, \\
& c_{7}=-\left(608-369 \gamma^{2}+810 \gamma^{4}\right) / 362880, \\
& c_{8}=\gamma\left(1288-489 \gamma^{2}+1890 \gamma^{4}\right) / 1935360, \\
& c_{9}=\left(9664-11061 \gamma^{2}+1296 \gamma^{4}-17010 \gamma^{6}\right) / 39191040 .
\end{aligned}
$$

With $r_{V}(\tau)$ from (A.3) and $a(\tau)$ from (A.4) and (A.6), the function $r_{M}(\tau)$ follows directly from the Friedmann Eq. (A.1b). The resulting expression is also a series,

$$
r_{M}(\tau)=\gamma \lambda \sum_{n=1}^{\infty} d_{n}(\gamma)(\sqrt{\lambda} \tau)^{n},
$$

where the coefficient $d_{n}$ for $n \geq 2$ is given by an expression involving the decay constant $\gamma$ and the previously determined coefficients $c_{n}, c_{n-1}, \ldots, c_{2}$. Specifically, the first eight coefficients are

$$
\begin{aligned}
& d_{1}=1, \quad d_{2}=-3 \gamma / 4, \\
& d_{3}=\left(-2+3 \gamma^{2}\right) / 6, \quad d_{4}=\gamma\left(6-5 \gamma^{2}\right) / 16, \\
& d_{5}=\left(64-207 \gamma^{2}+135 \gamma^{4}\right) / 720, \\
& d_{6}=-\gamma\left(28-37 \gamma^{2}+21 \gamma^{4}\right) / 192, \\
& d_{7}=\left(-608+4317 \gamma^{2}-3645 \gamma^{4}+1890 \gamma^{6}\right) / 30240, \\
& d_{8}=\gamma\left(3076-7770 \gamma^{2}+4995 \gamma^{4}-2430 \gamma^{6}\right) / 69120 .
\end{aligned}
$$

The coefficients $d_{n}$ for $\gamma \ll 1$ and $\gamma \gg 1$ are seen to effectively give alternating series. The same holds for coefficients $c_{n}$ from (A.6).

This completes the construction of a partial analytic solution of the ODEs (A.1), leaving for the future the rigorous determination of the convergence radius of the series appearing in (A.4) and (A.7). Still, the qualitative behavior of the solution can also be obtained numerically and has already been discussed in the second paragraph of Sec. 5. Comparing with the numerical solutions $a_{\text {num }}(\tau)$ and $r_{M, \operatorname{num}}(\tau)$ of the ODEs (A.1) for $\gamma \lesssim 1 / 10$, the convergence of the series in A.4 and A.7 is found to be rather poor, with a $\tau$ radius of convergence of order $1 / \sqrt{\lambda}$. 


\section{Note Added in Proof}

In order to clarify the last sentence of Sec. 1, it may be helpful to replace the energy density $\rho_{V}$ in the rest of the article by $\Lambda+\widetilde{\rho}_{V}$ and the dimensionless variable $r_{V}$ by $\lambda+\widetilde{r}_{V}$. Then, $\widetilde{\rho}_{V}$ and $\rho_{M}$ correspond, respectively, to the vacuum-type and standard-matter-type energy density of the produced particles. With $\gamma>0, k=0$, and $\widetilde{\rho}_{V}=\rho_{M}=0$ initially, the vacuum-type component $\widetilde{\rho}_{V}(t)$ from (9a) drops monotonically to the value $-\Lambda$, while the standard-matter-type component $\rho_{M}(t)$ first increases but then drops to zero due to the expansion of the Universe. Note that, for the simplest possible description of the instability of de Sitter-spacetime, it appears necessary to have at least two types of particle-production energy densities, distinguished by their equation of state.

\section{Acknowledgment}

It is a pleasure to thank A.A. Starobinsky and G.E. Volovik for valuable comments.

\section{References}

1. A.M. Polyakov, Nucl. Phys. B 797, 199 (2008), arXiv:0709.2899.

2. A.M. Polyakov, Nucl. Phys. B 834, 316 (2010), arXiv:0912.5503.

3. D. Krotov and A.M. Polyakov, Nucl. Phys. B 849, 410 (2011), arXiv:1012.2107.

4. E.T. Akhmedov, JHEP 1201, 066 (2012), arXiv:1110.2257.

5. Y.B. Zeldovich and A.A. Starobinsky, JETP Lett. 26, 252 (1977).

6. A.A. Starobinsky, JETP Lett. 30, 682 (1979).

7. S.W. Hawking and G.F.R. Ellis, The Large Scale Structure of Space-Time (Cambridge University Press, Cambridge, England, 1973).

8. G.W. Gibbons and S.W. Hawking, Phys. Rev. D 15, 2738 (1977).

9. F.R. Klinkhamer and G.E. Volovik, Phys. Rev. D 77, 085015 (2008), arXiv:0711.3170.

10. F.R. Klinkhamer and G.E. Volovik, Phys. Rev. D 78, 063528 (2008), arXiv:0806.2805.

11. R.M. Wald, General Relativity (Chicago University Press, Chicago, USA, 1984). 